\title{
Die Open-Access-Komponente in den DFG-geförderten Allianz-Lizenzen ${ }^{1}$
}

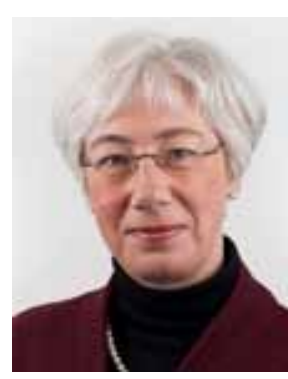

\author{
Kristine Hillenkötter \\ Leitung Informations- und Litera- \\ turversorgung - Zentrale Erwer- \\ bung und Erschließung \\ Niedersächsische Staats- und \\ Universitätsbibliothek \\ D-37070 Göttingen \\ E-Mail: \\ hillenkoetter@sub.uni-goettingen.de
}

The article gives a description and evaluation of the main aspects of these open access conditions, the outcomes of the negotiations and the challenges for all german research institutions, who have the opportunity to deal with these conditions now. Finally the Open Access clause of the alliance licenses has been compared with other activities for integrating Open Access conditions in national licenses on an international level.

Keywords: National licensing; alliance licenses; Open Access

\section{Hintergrund}

Die Allianz der deutschen Wissenschaftsorganisationen beInformation“ u.a. mit dem Handlungsfeld „Nationale Lizenzierung". Aufbauend auf den Erfahrungen mit den DFG-geförderten Nationallizenzen wurde in diesem Zusammenhang das Modell der DFG-geförderten Allianz-Lizenzen entwickelt und in den Verhandlungsrunden 2010 und 2011 erstmals umgesetzt: Für den Lizenzzeitraum 2011-2014 konnten 15 DFG-geförderte Allianz-Lizenzen für Zeitschriftenpakete abgeschlossen werden, die den für die Allianz-Lizenzen definierten Standards genügen, zu denen u.a. auch eine OpenAccess-Komponente gehört.

Die Entwicklung der Allianz-Lizenzen und deren wesentliche Merkmale werden kurz skizziert. Im Zentrum des Beitrags steht die Beschreibung der Open-Access-Komponente der Allianz-Lizenzen, die darauf bezogene Darstellung und Bewertung der ersten Verhandlungsergebnisse sowie die Chancen und Herausforderungen, die daraus für die wissenschaftlichen Einrichtungen in Deutschland abzuleiten sind. Abschließend erfolgt eine kurze Einbettung der Aktivitäten in den internationalen Kontext.

Schlüsselwörter: Nationale Lizenzierung; Allianz-Lizenzen; Open Access

\section{Open Access Conditions within the Alliance Li- censes funded by the by the German Research Foundation (DFG)}

National licensing belongs to the core activities of the Priority Initiative „Digital Information“ by the Alliance of German Science Organisations. Expanding the DFG's national licenses model the alliance license has been developed as a new type of DFG-funded national licenses. Alliance licenses have been negotiated firstly in 2010 and 2011. 15 DFG-funded alliance licenses for electronic journal packages could be finalized until then for the licensing period 2011-2014. They refer to defined standards, that require i.a. the grant of open access conditions.

1 Der Beitrag gründet sich auf zwei Vorträge zur gleichlautenden Thematik, die von der Verfasserin am 05.10.2011 im Rahmen der OpenAccess-Tage in Regensburg und am 24.05.2012 auf dem 101. Deutschen Bibliothekartag in Hamburg präsentiert wurden.
2008 wurde die Schwerpunktinitiative „Digitale Information" der Allianz der deutschen Wissenschaftsorganisationen gegründet $^{2}$, die für ihre Aktivitäten verschiedene Handlungsfelder definierte. Eines dieser Handlungsfelder ist der Bereich „Nationale Lizenzierung “3 ${ }^{\text {, }}$ der über nationale Lizenzabschlüsse und Aktivitäten im Bereich des Open Access eine möglichst umfassende Verfügbarkeit digitaler Publikationen erreichen soll. Dieser Anspruch wird gestützt durch die Open-Access-Policy der „Allianz-Initiative“. Mit der Umsetzung wurde eine Arbeitsgruppe beauftragt, die sich aus Vertretern der Max-Planck-Gesellschaft (MPG), der Helmholtz-Gemeinschaft (HGF), der Fraunhofer-Gesellschaft (FhG), der Leibniz-Gemeinschaft (WGL) und der Deutschen Forschungsgemeinschaft (DFG) sowie der Arbeitsgemeinschaft deutscher, österreichischer und schweizer Konsortien $(\mathrm{GASCO})^{4}$ zusammensetzt. Auch die verhandlungsführenden Einrichtungen der DFG-geförderten Nationallizenzen sind hier vertreten. ${ }^{5}$ Die Federführung liegt bei der Deutschen Forschungsgemeinschaft und der Helmholtz-Gemeinschaft. Auf der Grundlage der Erfahrungen mit den DFG-geförderten Nationallizenzen entwickelte die Allianz-AG „Nationale Lizenzierung" ${ }^{\prime \prime}$ das Konzept der Allianz-Lizenzen, für die hohe Standards und klare Verhandlungsziele definiert und 2010 im Rahmen der „Grundsätze für den Erwerb DFG-geförderter überregionaler Lizenzen" ${ }^{\text {"7 }}$ publiziert wur-

2 „Allianz-Initiative“, vgl. http://www.allianzinitiative.de/de/.

3 http://www.allianzinitiative.de/de/handlungsfelder/nationale_ lizenzierung/.

4 German, Austrian and Swiss Consortia Organisation (GASCO), vgl. http://www.hbz-nrw.de/angebote/digitale_inhalte/gasco/.

5 Bayerische Staatsbibliothek (BSB), Staatsbibliothek zu Berlin Preußischer Kulturbesitz (SBB), Technische Informationsbibliothek (TIB), Zentralbibliothek für Wirtschaftswissenschaften (ZBW), Zentralbibliothek für Medizin (ZB Med), Niedersächsische Staats- und Universitätsbibliothek Göttingen (SUB) und Universitätsbibliothek Johann Christian Senckenberg, Frankfurt am Main (UBF).

6 http://www.allianzinitiative.de/de/handlungsfelder/nationale_ lizenzierung/arbeitsgruppe/.

7 „Grundsätze“, vgl. http://www.dfg.de/download/programme/ wissenschaftliche_literaturversorgung_informationssysteme/ antragstellung/12_18/12_18.pdf. 
den. Sie werden ergänzt durch eine Musterlizenz zur Dokumentation der Lizenzabschlüsse. Inzwischen liegen aus den Jahren 2010 und 2011 erste Verhandlungsergebnisse und Vertragsabschlüsse der Allianz-Lizenzen für die Lizenzjahre 2011-2013 und 2012-2014 vor.

\section{Kennzeichen der Allianz-Lizenzen ${ }^{8}$}

Gegenstand der Allianz-Lizenzen sind die laufenden Inhalte von subskriptions- bzw. kostenpflichtigen elektronischen Zeitschriften, Datenbanken und E-Books ${ }^{9}$. Die Verhandlungen werden auf Basis der „Grundsätze für den Erwerb DFGgeförderter überregionaler Lizenzen "10 von den verhandlungsführenden Einrichtungen der DFG-geförderten Nationallizenzen geführt, deren Kreis Staatsbibliotheken, Zentrale Fachbibliotheken und Universitätsbibliotheken angehören ${ }^{11}$. Sie schließen für die Allianz-Lizenzen Mehrjahresverträge ab und organisieren zu deren Umsetzung nationale Opt-In-Konsortien als Angebot für die teilnahmeberechtigten Bibliotheken und wissenschaftlichen Einrichtungen in Deutschland. Die Kosten der DFG-geförderten Allianz-Lizenzen werden anteilig von den jeweiligen Konsortialteilnehmern und der DFG getragen, wobei der DFG-Förderanteil von den verhandlungsführenden Einrichtungen im Rahmen des DFGAntrags- und Begutachtungsverfahrens beantragt wird und bei positiver Begutachtung durch die DFG 25 Prozent der Gesamtkosten des Abschlusses umfasst ${ }^{12}$. Im Vergleich zu den überregionalen Angeboten der regionalen Konsortien in Deutschland zeichnen sich die Allianz-Lizenzen insbesondere durch erweiterte Nutzungsrechte wie das Recht auf lokales Hosting ${ }^{13}$, eine über eine Moving Wall verfügbar gemachte Archiv-Komponente ${ }^{14}$ sowie die Open-AccessKomponente aus (s.u.).

8 Vgl. auch http://www.nationallizenzen.de/ueber-nationallizenzen/ allianz-lizenzen-2011-ff.

9 Der Abschluss von Allianz-Lizenzen für abgeschlossene Datensammlungen ist grundsätzlich auf Basis der „Grundsätze für den Erwerb DFG-geförderter überregionaler Lizenzen“ möglich, steht jedoch derzeit nicht im Focus der Aktivitäten.

10 http://www.dfg.de/download/programme/wissenschaftliche_ literaturversorgung_informationssysteme/antragstellung/ 12_18/12_18.pdf.

11 Bayerische Staatsbibliothek (BSB), Staatsbibliothek zu Berlin Preußischer Kulturbesitzt (SBB), Technische Informationsbibliothek (TIB), Zentralbibliothek für Wirtschaftswissenschaften (ZBW), Zentralbibliothek für Medizin (ZB Med), Niedersächsische Staats- und Universitätsbibliothek Göttingen (SUB) und Universitätsbibliothek Johann Christian Senckenberg, Frankfurt am Main (UBF).

12 Auch im Rahmen des DFG-Antrags- und Begutachtungsprozesses nicht zur Förderung empfohlene Produkte dürfen unter dem Label „Allianz-Lizenz" angeboten werden, sofern sie vollumfänglich den in den „Grundsätzen für den Erwerb DFG-geförderter überregionaler Lizenzen "definierten Standards entsprechen. Die Finanzierung dieser Abschlüsse erfolgt dann zu 100 Prozent durch die Lizenzgebühren der am Konsortium partizipierenden Einrichtungen.

13 Hier verpflichten sich die Anbieter, die Daten auf Verlangen physisch an den Verhandlungsführer auszuliefern.

14 Nach Ablauf einer definierten Frist werden die Inhalte für alle wissenschaftlichen Einrichtungen in Deutschland dauerhaft verfügbar gemacht.

\section{Open-Access-Komponente der Allianz- Lizenzen}

Die Open-Access-Komponente der Allianz-Lizenzen ist in den „,Grundsätzen zum Erwerb DFG-geförderter überregionaler Lizenzen“ "sowie der Musterlizenz zur Dokumentation der erzielten Abschlüsse als Verhandlungsziel festgeschrieben. In den „Grundsätzen“ heißt es an entsprechender Stelle:

„Autoren aus autorisierten Einrichtungen sind ohne Mehrkosten berechtigt, ihre in den lizenzierten Zeitschriften erschienenen Artikel in der Regel in der durch den Verlag publizierten Form (z.B. PDF) zeitnah in ein (institutionelles oder disziplin-spezifisches) Repositorium ihrer Wahl einzupflegen und im Open Access zugänglich zu machen. Das gleiche Recht besitzen die autorisierten Einrichtungen, denen die jeweiligen Autoren angehören.Vereinbart werden kann auch, dass der Verlag es selbst übernimmt, Artikel von Autoren aus autorisierten Einrichtungen in ein Repositorium einzupflegen. "15

Damit werden konkrete Forderungen zu den zentralen Aspekten einer Veröffentlichung im Open Access formuliert:

- Berechtigung: Autoren und Institutionen

- Archivierbare Version: Verlagspublikation, z.B. PDF

- Embargofristen: „Zeitnahe“ Bereitstellung

- Bezugsmodalitäten: Verlagsbeteiligung möglich

- Repositorium: Freie Wahl (institutionell oder disziplinspezifisch)

Auf dieser Grundlage können in Deutschland jetzt erstmals Open-Access-Klauseln in konsortiale Lizenzverträge für subskriptionspflichtige Inhalte integriert werden ${ }^{16}$. Postuliert wird das Zweitverwertungsrecht der in den Subskriptionszeitschriften primär publizierten Veröffentlichungen im Sinne des ,Self-Archiving“. Der Ansatz verweist damit auf den grünen Weg des Open Access und ergänzt die hierzulande bereits verbreitete Praxis des Abschlusses von lokalen Hybrid-Verträgen für subskriptionspflichtige Inhalte ${ }^{17}$. Die in Deutschland ebenfalls bereits aktive Praxis lokaler Vertragsabschlüsse mit Open-Access-Verlagen nach Maßgabe des goldenen Weges steht dem gegenüber.

Die Open-Access-Komponente der Allianz-Lizenzen kann zwar auch für Volltextdatenbanken und E-Book-Angebote verhandelt werden, bezieht sich jedoch in erster $\mathrm{Li}$ nie auf Zeitschriftenliteratur. Für Zeitschriftenangebote ist

15 „Grundsätze für den Erwerb DFG-geförderter überregionaler Lizenzen“, D23. Stand: Juni 2010. Vgl. http://www.dfg.de/download/ programme/wissenschaftliche_literaturversorgung_informationssysteme/ antragstellung/12_18/12_18.pdf.

16 Einzige Ausnahme bildet der nationale Konsortialvertrag der GASCO (German, Austrian and Swiss Consortia Organisation, http://www. hbz-nrw.de/angebote/digitale_inhalte/gasco/) mit dem Open-AccessVerlag BioMedCentral.

17 Hybride Geschäftsmodelle greifen für die Finanzierung von Publikationen teils auf Subskriptionsgebühren, teils auf Publikationsgebühren zurück. Nach Maßgabe von lokal geschlossenen Hybrid-Verträgen zu einem subskriptionspflichtigen Zeitschriftenpaket können alle Autoren der lizenznehmenden Einrichtung ihre in den betreffenden Zeitschriften konventionell publizierten Artikel gegen Zahlung einer definierten Publikationsgebühr online entgeltfrei im Open Access zugänglich machen. Dabei kann die Publikationsgebühr auch zentral durch die lizenznehmende Einrichtung abgegolten werden. 
die Gewährung dieses Nutzungsrechtes durch den Anbieter unabdingbare Voraussetzung für die DFG-Förderung von Abschlüssen entsprechender Allianz-Lizenzen. Insofern wird die Open-Access-Komponente der Allianz-Lizenzen durch die Open-Access-Policies der DFG und der AllianzInitiative gestützt ${ }^{18}$.

Hinsichtlich der einzelnen Aspekte des Nutzungsrechtes sowie der dafür geforderten Kostenneutralität und des Hinweises, die Verlage in den sich anschließenden Workflow einzubeziehen, geht der Anspruch der Open-Access-Komponente deutlich über die derzeit in SHERPA/ROMEO formulierten Standards für das Self-Archiving ${ }^{19}$ hinaus: Sie soll die Anbieter zur Verbesserung ihrer Open-Access-Konditionen bewegen.

\section{Erste Ergebnisse 2011-2014}

In den Jahren 2010 und 2011 wurden Allianz-Lizenzen für insgesamt 19 Produkte abgeschlossen, darunter eine E-BookCollection, 3 Datenbanken und 15 Zeitschriftenangebote, die optimal den Anforderungen der „Grundsätze für den Erwerb DFG-geförderter überregionaler Lizenzen“ entsprechen. Demzufolge werden im Rahmen der Allianz-Lizenzen derzeit folgende Zeitschriftenpakete mit Open-Access-Komponente angeboten:

- AIP Journals (AIP)

- Annual Reviews (AR)

- Berkley Electronic Press Academic Journals (Bepress)

- BMJ Journal Online Collection (BMJ)

- Cambridge University Press (CUP)

- China Academic Journals (CAJ)

- EMS Journals (EMS)

- Karger Journals (Karger)

- Oxford Journals Current (OUP)

- RSC Journals (RSC)

- Sage Journals online (Sage)

- De Gruyter Online Zeitschriften / Geistes- u. Sozialwissenschaften (WdG)

- De Gruyter Online Zeitschriften / Geistes- u. Sozialwissenschaften Ergänzung (WdG, keine Förderung ${ }^{20}$ )

- De Gruyter Online Zeitschriften / STM (WdG, keine Förderung ${ }^{21}$ )

18 http://www.allianzinitiative.de/de/handlungsfelder/open_access/, http://www.dfg.de/dfg_magazin/forschungspolitik_standpunkte_ perspektiven/open_access/index.html, http://www.dfg.de/download/ pdf/foerderung/programme/lis/pos_papier_elektron_publizieren_ 0504.pdf und http://www.dfg.de/dfg_magazin/forschungspolitik_ standpunkte_perspektiven/open_access/gefoerderte_projekte/ index.html.

19 http://www.sherpa.ac.uk/romeo/specialpagelist.php?la=en\&fIDnum $=\mid \&$ mode $=$ simple.

20 Das Angebot entspricht in den Konditionen exakt dem DFG-geförderten Paket für die Geistes- und Sozialwissenschaften, kann jedoch aus lizenzrechtlichen Gründen während der aktuellen Vertragslaufzeit nicht inhaltlich mit diesem verschmolzen werden. Eine Verknüpfung mit dem Grundangebot ist im Rahmen der Erneuerung für die nächste Lizenzperiode 2014-16 geplant.

21 Das Angebot entspricht in den Konditionen exakt dem DFG-geförderten Paket für die Geistes- und Sozialwissenschaften, erhielt jedoch bei Antragstellung in 2010 aus fachlichen Gründen keine DFG-Förderung.
- De Gruyter Online Zeitschriften / STM Ergänzung (WdG, keine Förderung ${ }^{22}$ )

In den genannten Abschlüssen konnten die einzelnen Aspekte der Open-Access-Komponente wie folgt durchgesetzt werden $^{23}$ :

\section{- Berechtigung:}

Nahezu alle Angebote erteilen die Berechtigung zum vertraglich vereinbarten Zweitverwertungsrecht an Autoren und Institutionen (13 Angebote), die beiden übrigen beschränken sich auf die Autoren. Der Begriff des „Autors“ bezieht sich dabei sowohl auf den ,,corresponding author“ als auch auf den ,contributing author“"

\section{- Archivierbare Version:}

Alle Angebote bieten die geforderte Verlagspublikation als archivierbare Version an, womit in nahezu allen Fällen das Verlags-PDF (14 Angebote) und nur in einem Fall das Postprint im Sinne einer Post-Peer-Reviewed Version gemeint ist.

- Embargofristen:

8 Angebote, also mehr als 50 Prozent der Fälle verzichten komplett auf die Festlegung einer Embargofrist und entsprechen damit vollumfänglich dem Anspruch der „zeitnahe(n)“ Bereitstellung. In allen anderen Fällen werden Embargofristen in sehr unterschiedlicher Größenordnung verhängt, die zwischen 6 und 36 Monaten liegen. Die durchschnittliche Embargofrist beträgt über alle Angebote kalkuliert 7 Monate.

\section{- Bezugsmodalitäten:}

Die meisten Angebote gewähren den Direktdownload der Artikel aus der Datenbank (13 Angebote), und 2 Verlage stellen die Artikel auf Anfrage beim Verlag zur Verfügung.

- Repositorium:

13 Angebote stellen den Autoren und Institutionen die freie Wahl eines institutionellen oder disziplinspezifischen Repositoriums zur Archivierung der Artikel anheim. Die Ausnahme bilden 2 Angebote, die die Archivierung jeweils auf ein institutionelles bzw. ein verlagseigenes Repositorium festlegen.

Ein Vergleich der erzielten Verhandlungsergebnisse mit den bei Vertragsabschluss eingetragenen Open-Access-Konditionen der betreffenden Anbieter in SHERPA/ROMEO ${ }^{24}$ macht deutlich, dass in den ersten beiden Verhandlungsrunden der Allianz-Lizenzen nicht nur die Standards der „Grundsätze

22 Das Angebot entspricht in den Konditionen exakt dem DFG-geförderten Paket für die Geistes- und Sozialwissenschaften. Eine Verknüpfung mit dem Grundangebot STM ist im Rahmen der Erneuerung für die nächste Lizenzperiode 2014-16 geplant.

23 Eine detaillierte Übersicht mit den pro Angebot und Produkt verhandelten Konditionen findet sich auf der Website der DFG-geförderten Lizenzen für elektronische Medien (vgl. http://www.nationallizenzen. de/open-access) Die Produktübersicht wird laufend aktualisiert. Sie gibt auch Auskunft über DFG-geförderte Nationallizenzen, für die in den Jahren 2009 bis 2010 Open-Access-Konditionen mitverhandelt wurden, sowie über nicht DFG-geförderte nationale Lizenzen aus dem Verhandlungskontext der Allianz-Lizenzen, die jedoch vollumfänglich den ,Grundsätzen zum Erwerb DFG-geförderter überregionaler Lizenzen" entsprechen und damit ebenfalls eine Open-Access-Komponente enthalten.

24 Vgl. http://www.dini.de/wiss-publizieren/sherparomeo/. 
für den Erwerb DFG-geförderter überregionaler Lizenzen“" durchgesetzt, sondern in der überwiegenden Anzahl der Fälle auch die etablierten Open-Access-Konditionen der betroffenen Verlage optimiert werden konnten. Die Ursachen für diesen Erfolg dürfen in der konsequenten Stützung der Verhandlungen durch die Open-Access-Policies der AllianzInitiative und der $\mathrm{DFG}^{25}$ sowie in der mit den Allianz-Lizenzen einhergehenden Verhandlungspraxis im Sinne einer Bündelung des Verhandlungspotentials auf der Nachfrageseite gesehen werden.

\section{Nutzung durch wissenschaftliche Einrichtungen in Deutschland}

Die im Rahmen der Allianz-Lizenzen verhandelten OpenAccess-Konditionen stehen jetzt zur Nutzung durch die wissenschaftlichen Einrichtungen in Deutschland bereit. Nutzungsberechtigt sind alle Institutionen, die an einer der Lizenzen teilnehmen, sowie die Autoren, die mit diesen Einrichtungen verbunden sind. Autoren und Einrichtungen, die die bereitgestellten Open-Access-Konditionen der AllianzLizenzen nutzen wollen, sehen sich dabei mit vielfältigen Herausforderungen konfrontiert.

Diese liegen zum einen in der Komplexität der verhandelten Rechte begründet. Beispielsweise kann es unterschiedliche Konditionen für ein Zeitschriftenpaket geben. Daher sind die produktspezifischen Konditionen zunächst mit Blick auf die Anforderungen und Möglichkeiten der eigenen Einrichtung zu analysieren.

Zum anderen gilt es dann, die Artikel aus den lizenzierten Zeitschriftenpaketen zu identifizieren, für die jeweils eine Berechtigung zur Nutzung des Zweitverwertungsrechtes besteht. Im Bemühen herauszufinden, ob und was Autoren, die einer an der betreffenden Allianz-Lizenz partizipierenden Einrichtung angehören, in den lizenzierten Inhalten publiziert haben, und darüber festzustellen, für welche Inhalte an der betreffenden Einrichtung überhaupt die Berechtigung zur Nutzung der Open-Access-Konditionen besteht (, ,affiliated articles"), müssen die Websites oder konkrete Ansprechpartner der Verlage, aber auch interne sowie externe und z.T. kommerzielle Forschungsdatenbanken wie z.B. Web of Science konsultiert werden.

Im nächsten Schritt ist für die Beschaffung der auf diesem Weg identifizierten Publikationen zu sorgen, die über den Direktdownload aus dem Verlagsangebot durch die Einrichtung selbst oder auf Anfrage der Einrichtung durch Lieferung vom Verlag oder Autor erfolgen kann.

Bei der sich anschließenden Archivierung der Publikation in den dafür ausgewählten Repositorien sind Embargofristen für die Freischaltung zu beachten. Und schließlich führt

25 http://www.allianzinitiative.de/de/handlungsfelder/open_access/, http://www.dfg.de/dfg_magazin/forschungspolitik_standpunkte_ perspektiven/open_access/index.html, http://www.dfg.de/download/ pdf/foerderung/programme/lis/pos_papier_elektron_publizieren_ 0504.pdf und http://www.dfg.de/dfg_magazin/forschungspolitik_ standpunkte_perspektiven/open_access/gefoerderte_projekte/index. html. auch die Verfügbarmachung und Bereitstellung der Publikation im Open Access über die Repositorien wieder zu neuen Herausforderungen, denn die Nutzung der auf diese Weise frei verfügbar gemachten Publikation darf natürlich über das Repositorium nur im Rahmen der Nutzungsbedingungen der zugehörigen Allianz-Lizenz erfolgen. Diesen Sachverhalt gilt es durch die Betreiber der Repositorien zu kommunizieren; die Verantwortung für die Einhaltung der Nutzungsbedingungen liegt bei den lizenznehmenden Einrichtungen.

Um die Umsetzung der Open-Access-Komponente der Allianz-Lizenzen in die Praxis zu befördern und die Autoren und Einrichtungen bei der Nutzung der verhandelten Rechte zu unterstützen, stehen eine tabellarische Übersicht der einzelnen Produkte und Konditionen sowie im Sinne einer praktischen Anleitung eine Handreichung für RepositoryManager, Bibliothekare und Autoren zur Verfügung ${ }^{26}$. Beide Dokumente sind im Kontext der Arbeitsgruppen „Nationale Lizenzierungen“ und „Open Access“ der Allianz-Initiative entstanden und über deren Website sowie die der DFG-geförderten Lizenzen für elektronische Medien zugänglich. ${ }^{27}$ Darüber hinaus können technische Lösungen und Workflows hilfreich sein, die in verschiedenen Projektkontexten entwickelt wurden und jetzt zur Nachnutzung bereit stehen (z.B. SWORD-Technologie, Szenarien für Workflows und den Aufbau von Infrastrukturen aus den Projekten PEER und OpenAIRE ${ }^{28}$ ).

Trotz der skizzierten Herausforderungen überwiegt der Wert der im Rahmen der Allianz-Lizenzen verhandelten Zweitverwertungsrechte für die lizenznehmenden Einrichtungen: Sie erschließen den wissenschaftlichen Einrichtungen in Deutschland kostenfrei weitere Quellen zur Befüllung der institutionellen und disziplinspezifischen Repositorien mit qualitativ hochwertigen wissenschaftlichen Inhalten. Damit dienen sie der Stützung der Open-Access-Policies in den einzelnen Einrichtungen und der Verbreitung von Open Access im Allgemeinen.

\section{Internationaler Vergleich ${ }^{29}$}

Auch in anderen Ländern gibt es seit einigen Jahren im Kontext nationaler Lizenzierungen subskriptionspflichtiger (Zeitschriften-)Inhalte Bestrebungen zur Integration von Open-Access-Komponenten.

Am weitesten ist die Entwicklung im anglo-amerikanischen Raum fortgeschritten, wo bereits entsprechende

26 Open-Access-Rechte in Allianz- und Nationallizenzen. Eine Handreichung für Repository - Manager, Bibliothekare und Autoren. Hrsg.: Arbeitsgruppen „Nationale Lizenzierung“ und „Open Access“ der Schwerpunktinitiative „Digitale Information“ der Allianz der deutschen Wissenschaftsorganisationen. April 2012. http://dx.doi. org/10.2312/allianzoa.004.

27 http://www.nationallizenzen.de/open-access und http://dx.doi. org/10.2312/allianzoa.004.

28 http://swordapp.org/, http://www.peerproject.eu/, http://www. openaire.eu/.

29 Vgl. hierzu auch: Birgit Schmidt, Kathleen Shearer: Licensing Revisited: Open Access Clauses in Practice. Eingereicht bei LIBER Quarterly, Mai 2012. 
Rechte in den Lizenzverträgen verankert werden. So hat die amerikanische Association of Research Libraries 2009 eine model language zur Fixierung von Autorenrechten in Lizenzverträgen formuliert ${ }^{30}$, das zentrale Konsortium JISC in Großbritannien schreibt die Verfügbarmachung von Vollexten auf Repositorien und das Monitoring von Hybridmodellen ab 2009 im Rahmen der NESLi2 model license fest ${ }^{31}$.

In Frankreich werden seit 2011 Klauseln zur Selbstarchivierung der lizenzierten Inhalte sowie zu weiteren organisatorischen Aspekten, wie z.B. der Wahl des Repositoriums, der Embargofristen, der archivierbaren Version etc., in die Verhandlungsrichtlinien des zentralen nationalen Konsortiums Couperin ${ }^{32}$ integriert, auch Schweden bezieht seit 2011 vor dem Hintergrund der Open-Access-Policy der schwedischen Nationalbibliothek Open-Access-Komponenten in die Lizenzverhandlungen mit Verlagen ein $^{33}$.

Polen und die Niederlande experimentieren mit nationalen Abschlüssen von Hybridverträgen mit Springer OpenChoice und SAGE Choice ${ }^{34}$.

Im Vergleich zu diesen Entwicklungen verbinden die deutschen Allianz-Lizenzen seit 2010 diese verschiedenen Ansätze und Merkmale:

- Bezug auf die Open-Access-Policies von Einrichtungen und Bewegungen, die für das Themenfeld „Nationale Lizenzierung“" von zentraler, impulsgebender Relevanz sind.

- Integration von Open-Access-Komponenten in definierte Verhandlungsrichtlinien und -standards für die nationale Lizenzierung subskriptionspflichtiger Inhalte.

30 http://authorrights. wordpress.com/.

31 http://www.jisc-collections.ac.uk/nesli2/NESLi2-Model-Licence-/.

32 http://www.couperin.org/.

33 http://www.kb.se/english/about/news/New-Open-Access-policy/.

34 Niederlande: Nationalvertrag des UKB (http://www.ukb.nl/index. html) mit Springer OpenChoice (2007-2012) und Sage Choice (seit 2012), vgl. http://www.openaccess.nl/index.php/news/302-dutchoa-licensing-news. Polen: Nationalvertrag der Universität Warschau mit Springer OpenChoice (seit 2010), vgl. http://www.eifl.net/news/ national-program-springer-open-choiceopen-acc.
- Integration von Open-Access-Klauseln in eine Musterlizenz im Rahmen eines neuen Lizenzmodells für die nationale Lizenzierung subskriptionspflichtiger Inhalte.

- Verhandlungen und Abschlüsse nationaler Lizenzen mit Open-Access-Komponenten für das Self-Archiving. Damit erweist sich die Open-Access-Komponente der DFGgeförderten Allianz-Lizenzen auch im internationalen Kontext als innovativ und gut positioniert.

\section{Zusammenfassung und Ausblick}

Die Open-Access-Komponente der DFG-geförderten Allianz-Lizenzen bildet ein wesentliches Merkmal dieses neuen Lizenztyps für die nationale Lizenzierung in Deutschland. Sie wird strategisch gestützt durch die Open-Access-Policies der DFG und der Allianz-Initiative sowie durch definierte Verhandlungsstandards, die Bündelung des Verhandlungspotenzials auf der Nachfrageseite und die Verankerung in einer Musterlizenz. Postuliert wird das Recht zur kostenlosen Zweitveröffentlichung von lizenzierten Inhalten durch die berechtigten Autoren und Einrichtungen nach definierten Parametern im Sinne des Self-Archiving auf dem grünen Weg des Open Access. Erste Verhandlungsergebnisse in 2010 und 2011 zeigen, dass bei vielen Verlagen auf diese Weise eine Verbesserung ihrer etablierten Open-Access-Konditionen erreicht werden konnte. Den wissenschaftlichen Einrichtungen in Deutschland erschlieBen sich über die Open-Access-Komponente der DFG-geförderten Allianz-Lizenzen kostenfrei weitere Quellen zur Befüllung der Repositorien mit hochwertigen Inhalten. Dadurch werden die Open-Access-Strategien der Einrichtungen unterstützt und die allgemeine Verbreitung von Open Access gefördert. Auch im internationalen Kontext erweist sich diese Entwicklung als innovativ und gut positioniert. Es bleibt zu wünschen, dass die hier verhandelten Rechte und Möglichkeiten umfänglich von den wissenschaftlichen Einrichtungen in Deutschland genutzt und in Anspruch genommen werden. 